\title{
Current Directions and Trends of Cancer Chemotherapy: An Overview of the Recently Approved Small-Molecules Anticancer Drugs in the Past Decade
}

\author{
Hadeer M Soudan ${ }^{1,2}$, Asmaa A Abdel Haseeb ${ }^{1,3}$, Ahmed A Nasser $^{1,4}$ and Sherif F Hammad ${ }^{1,5 *}$ \\ ${ }^{1}$ Basic and Applied Sciences Institute, Egypt-Japan University of Science and Technology (E-JUST), New Borg El-Arab City, 21934 Alexandria, Egypt \\ ${ }^{2}$ Department of Biotechnology, Institute of Graduate Studies and Research (IGSR), Alexandria University, Alexandria, Egypt \\ ${ }^{3}$ Chemistry Department, Faculty of Science, Minia University, El Minya, Egypt
}

${ }^{4}$ Pharmaceutical Medicinal Chemistry \& Drug Design Department, Faculty of Pharmacy (Boys), Al-Azhar University, Cairo 11884, Egypt

${ }^{5}$ Pharmaceutical Chemistry Department, Faculty of Pharmacy, Helwan University, Ain Helwan 11795, Cairo, Egypt

*Corresponding author: Sherif F Hammad, Associate Professor of Medicinal Chemistry, Egypt-Japan University of Science and Technology, Egypt.

Dedication to Professor. Forrest Smith, Harrison School of Pharmacy, Auburn University, Alabama, USA.

To Cite This Article: Hadeer M Soudan, Asmaa A Abdel Haseeb, Ahmed A Nasser, Sherif F Hammad. Current Directions and Trends of Cancer Chemotherapy: An Overview of the Recently Approved Small-Molecules Anticancer Drugs in the Past Decade. Am J Biomed Sci \& Res. 2021 13(4). AJBSR.MS.ID.001888. DOI: 10.34297/AJBSR.2021.13.001888.

Received: 㘹 June 28, 2021; Published: 眥 July 14, 2021

\begin{abstract}
Background: The everlasting challenge to develop more potent, more selective and less toxic chemotherapeutic antitumor drugs constitutes a substantial weapon to defeat the continuous multidrug resistance characteristics of many cancers. Therefore, the need for the discovery and development of unprecedented antineoplastics drugs still exists.

Results: In this mini review a survey of the novel targets and trends in cancer chemotherapy has been investigated to explore the potential therapeutic lines and treatment protocols. 70 novel small-molecule antineoplastic were studied and categorized according to their mode of action, medicinal use, year of FDA-approval as well as chemical structures. The vast majority of these recently approved drugs over the past decade are kinase inhibitors while the hormone antagonists represent the minority of these novel drugs. The two years of 2018 and 2020 represent the years of the highest approval numbers of small-molecules anticancer drugs with 10 approved drugs, while the year of 2016 is the one with the least number of just 2 newly approved drugs.
\end{abstract}

Conclusion: In this study, a quick and yet comprehensive survey has been conducted to explore the major targets and trends in the last decade with respect to small-molecule anticancer drugs that have been recently FDA-approved. A new approach of classification of these drugs based on their detailed mechanism of action was demonstrated.

Keywords: Anticancer Drugs; Tyrosine Kinase Inhibitors; Cancer-Drug-Targets

\section{Introduction}

Cancer as a broad expression of various malignancies remains as a global health concern as it accounts for more than 10 million annual mortalities throughout the past few years representing about $14.6 \%$ of deaths globally. Therefore, Cancer as a major health problem and a life-threatening disease stands out as the second leading cause of death after cardiovascular diseases [1]. The exact root causes and underlying reasons specific for variable cancer illnesses remain undefined and hence the discovery and development of novel antineoplastics to face the continuous mutations and multi-drug resistance is still pivotally demanded. Multiple classification systems have been postulated such as antimetabolites and alkylating agents, also they could be broadly categorized into targeted drugs and cytotoxic agents as another system [2]. Herein, a more comprehensive type of classification 
based on the detailed mechanism of action could be investigated via exploring the main novel trends of the recently approved anticancer drugs. This minireview includes only the FDA approved small molecules anticancer drugs from 2011 to 2020. Despite being apart from the scope of the current review, it is worthy to mention that the passing decade witnessed the dawn of cancer immunotherapy via the inhibition of the regulation of the adaptive immune response against malignancy. The prototype of this class Ipilimumab was approved in 2011 as an immune checkpoint inhibitor of cytotoxic T-lymphocyte-associated antigen 4 (CTLA-4), for the treatment of melanoma [3]. The focus of this review is substantially directed to small-molecules anticancer drugs since they constitute a major segment of all the approved small molecules in $>26 \%$ ratio and in $>17 \%$ of all approved drugs (with all categories that include biologicals and biopharmaceuticals), as represented in Table 1.

\begin{tabular}{|c|c|c|c|c|c|c|c|c|c|c|c|}
\hline & 2011 & 2012 & 2013 & 2014 & 2015 & 2016 & 2017 & 2018 & 2019 & 2020 & Last decade \\
\hline Total approved drugs & 30 & 39 & 27 & 41 & 45 & 22 & 46 & 59 & 48 & 53 & 410 \\
\hline Small molecule drugs & 22 & 25 & 21 & 25 & 30 & 8 & 31 & 37 & 32 & 35 & $\begin{array}{c}266 \\
64.90 \%\end{array}$ \\
\hline $\begin{array}{c}\text { Small molecules anticancer } \\
\text { drugs }\end{array}$ & 5 & 9 & 5 & 4 & 9 & 2 & 8 & 10 & 8 & 10 & $\begin{array}{c}70 \\
17 \%\end{array}$ \\
\hline
\end{tabular}

\section{Discussion}

Trending of different classes of small molecules anticancer drugs in the past decade depending on the mode of action of the FDAapproved drugs could be categorized according to their mode of action. Figures 1 and 2 illustrate the outline of the classification and the proportions of small molecules anticancer drugs, respectively.
The drugs are classified into five major classes: kinases inhibitors, drugs affecting nucleic acids, antiandrogens, hedgehog pathway inhibitors and miscellaneous.

In addition, Figures 3-8 illustrate the chemical structures of all the mentioned drugs with the year of FDA approval and brief about their uses and modes of action. These recently approved drugs could be classified as following:
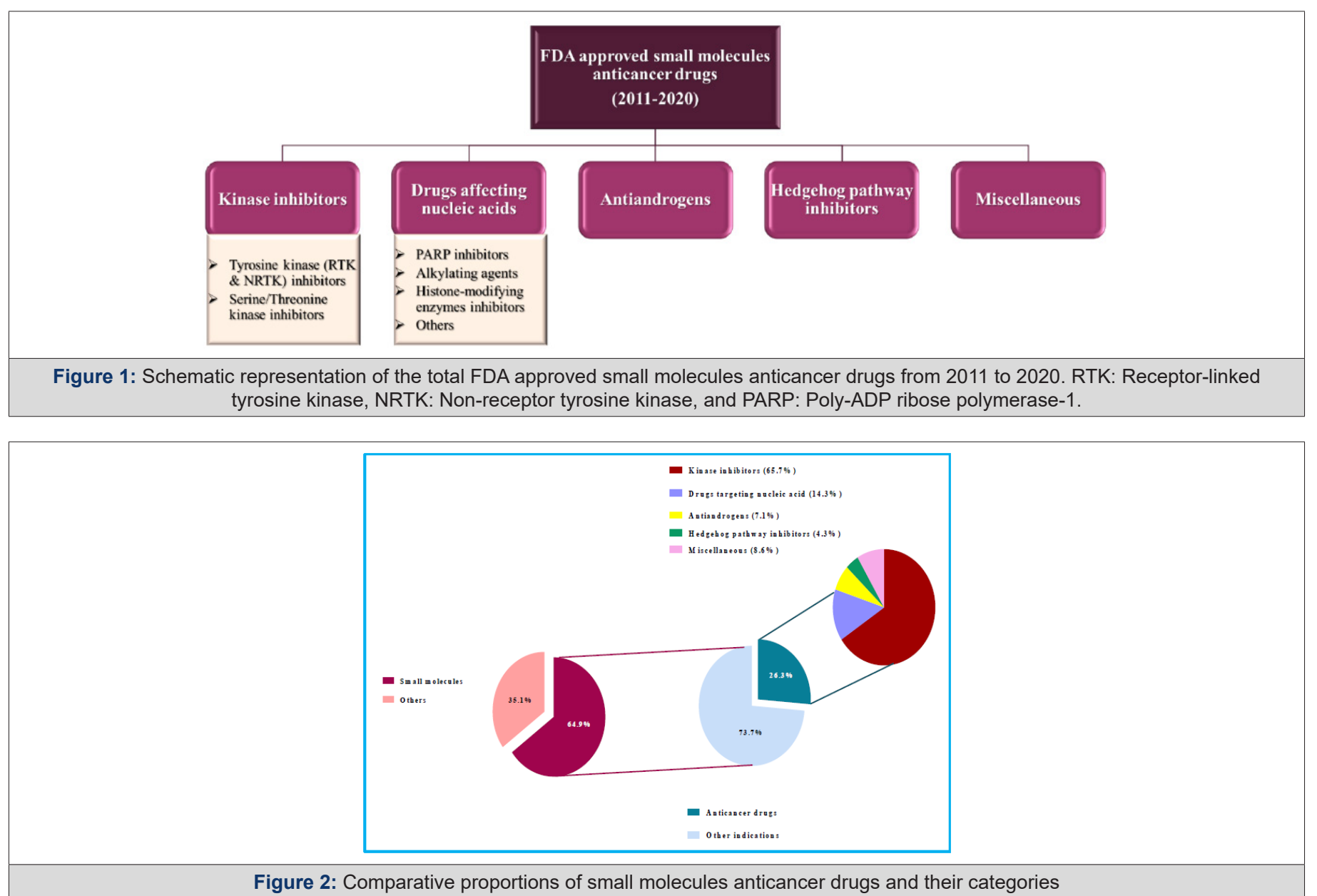
<smiles>COc1cc2c(Nc3ccc(Br)cc3F)ncnc2cc1OCC1CCN(C)CC1</smiles>

Vandetanib, Metastatic medullary thyroid cancer,VEGF, EGFR and RET inhibitor,(2011)

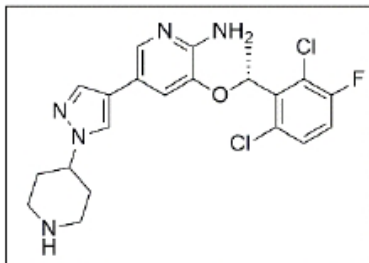

Crizotinib, metastatic non-small cell lung cancer (NSCLC), protein kinase inhibitor, (2011)
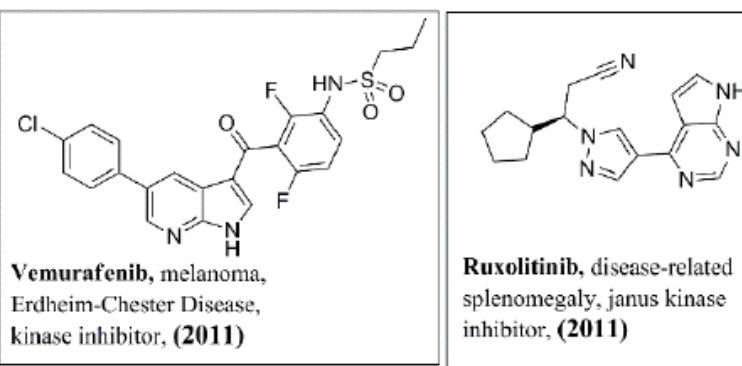

Ruxolitinib, disease-related splenomegaly, janus kinase inhibitor, (2011)

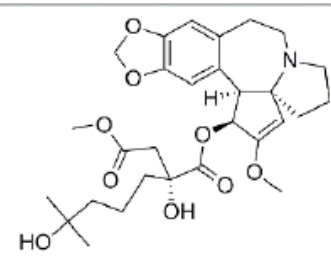

Omacetaxine mepesuccinate,

chronic myeloid leukaemia, protein translation inhibitor, (2012)<smiles>CS(=O)(=O)c1ccc(C(=O)Nc2ccc(Cl)c(-c3ccccn3)c2)c(Cl)c1</smiles>

Vismodegib, Metastatic or locally advanced basal cell carcinoma, Smoothened (Hedgehog pathway) inhibitor, (2012)

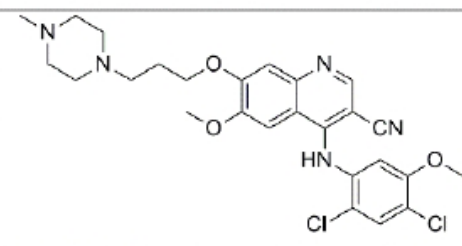

Bosutinib, Chronic, accelerated or blast phase $\mathrm{Ph}+$ chronic myelogenousleukaemia BCR-ABL inhibitor; SRC family kinase inhibitor, (2012)

Regorafenib, Metastatic
colorectal cancer, Multikinase
inhibitor with targets including
RET, VEGFR1, -2, -3 and KIT,
(2012)<smiles>COc1cc2nccc(Oc3ccc(NC(=O)C4(C(=O)Nc5ccc(F)cc5)CC4)cc3)c2cc1OC</smiles>

Cabozantinib, Progressive, metastatic medullary thyroid cancer Multikinase inhibitor with targets including RET, MET, VEGFR1, -2, -3, KIT and FLT3, (2012)

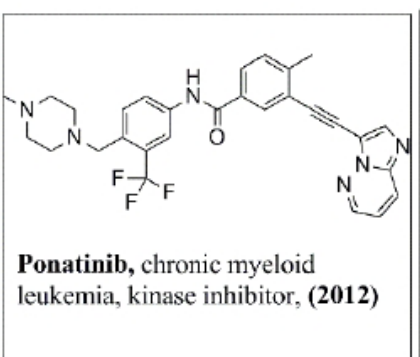

Enzalutamide, Prostate cancer, androgen receptor inhibitor, (2012)

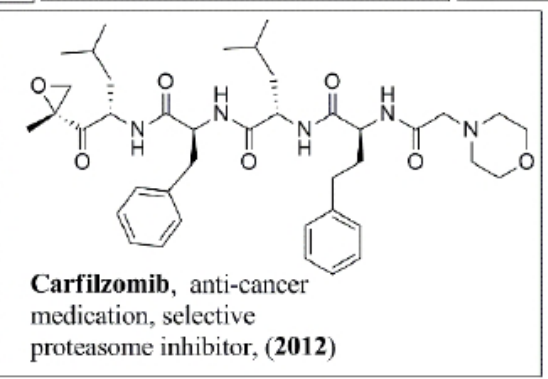

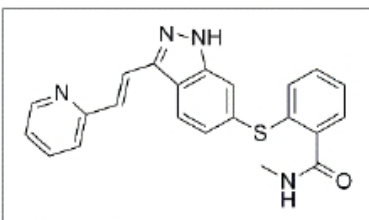

Axitinib,

renal cell carcinoma, VEGFR and kinase inhibitor, (2012)

Figure 3: The chemical Structures of the approved drugs from 2011 to 2012. 
<smiles>CC(=O)Nc1cccc(-n2c(=O)n(C3CC3)c(=O)c3c(Nc4ccc(I)cc4F)n(C)c(=O)c(C)c32)c1</smiles>

Trametinib, Unresectable or metastatic melanoma with BRAFV600E mutation, MEK1 and MEK2 kinase inhibitor,(2013)<smiles>CC(C)(C)c1nc(-c2cccc(NS(=O)(=O)c3c(F)cccc3F)c2F)c(-c2ccnc(N)n2)s1</smiles>

Dabrafenib, melanoma, non-small cell lung cancer, and thyroid cancer, B-Raf inhibitor, (2013)

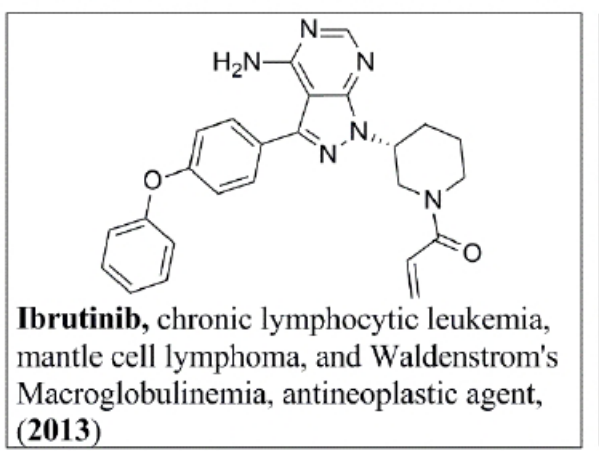<smiles>CN(C)C/C=C/C(=O)Nc1cc2c(Nc3ccc(F)c(Cl)c3)ncnc2cc1OC1CCOC1</smiles>

Afatinib, metastatic NSCLC whose tumours have EGFR exon 19 deletions or exon 21 (L858R) substitution mutations, kinase inhibitor, (2013)<smiles>Nc1cccc2c1C(=O)N(C1CCC(=O)NC1=O)C2=O</smiles>

Pomalidomide, multiple myeloma,

inhibits angiogenesis and myeloma cell growth, (2013)<smiles>Cc1cc(Nc2ncc(Cl)c(Nc3ccccc3S(=O)(=O)C(C)C)n2)c(OC(C)C)cc1C1CCNCC1</smiles>

Ceritinib, ALK-positive metastatic NSCLC, Kinase inhibitor against ALK, IGF1R, insulin receptor and ROS1, (2014)<smiles>CCC(Nc1ncnc2nc[nH]c12)c1nc2cccc(F)c2c(=O)n1-c1ccccc1</smiles>

Idelalisib, certain blood cancers. phosphoinositide 3-kinase inhibitor, (2014)<smiles>O=C(/C=C/c1cccc(S(=O)(=O)Nc2ccccc2)c1)NO</smiles>

Belinostat, hematological malignancies and solid tumors, histone deacetylase inhibitor, (2014)<smiles>O=C(c1cc(Cc2n[nH]c(=O)c3ccccc23)ccc1F)N1CCN(C(=O)C2CC2)CC1</smiles>

Olaparib, Advanced BRCAmutated ovarian cancer, PARP inhibitor,(2014) 

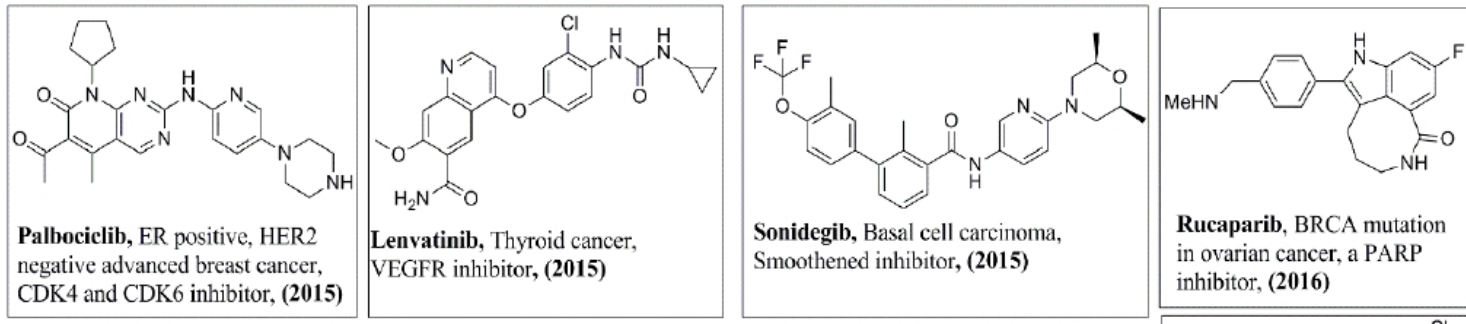

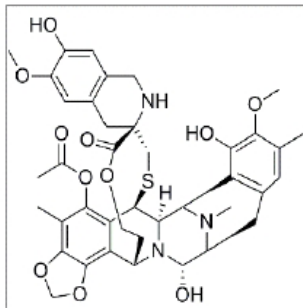

Trabectedin,Liposarcoma or

leiomyosarcoma, Alkylating drug (2015)

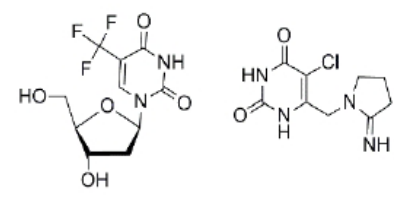

Trifluridine and tipiracil, colorectal cancer or gastric cancer, nucleoside metabolic inhibitor, and a thymidine phosphorylase inhibitor, (2015)
(E)

Panobinostat, multiple myeloma, nonselective histone deacetylase inhibitor, (2015)

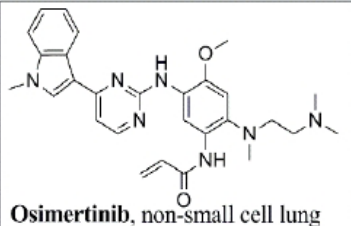
(2015)
Osimertinib, non-small cell lung
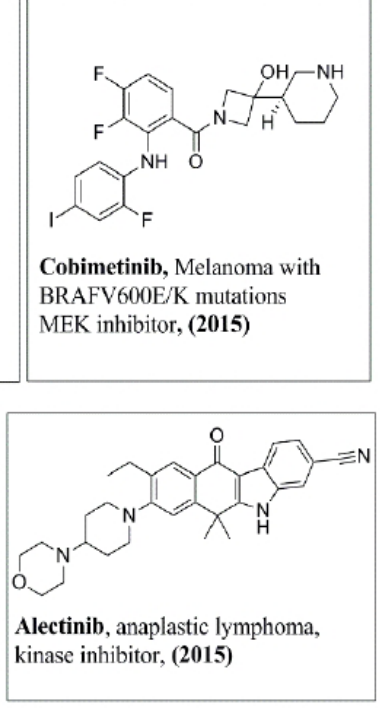

Rucaparib, BRCA mutation in ovarian cancer, a PARP inhibitor, (2016)

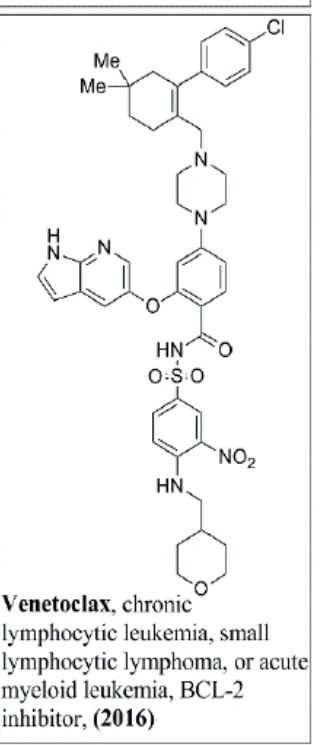

Figure 5: The chemical structures of the approved drugs from 2015 to 2016.
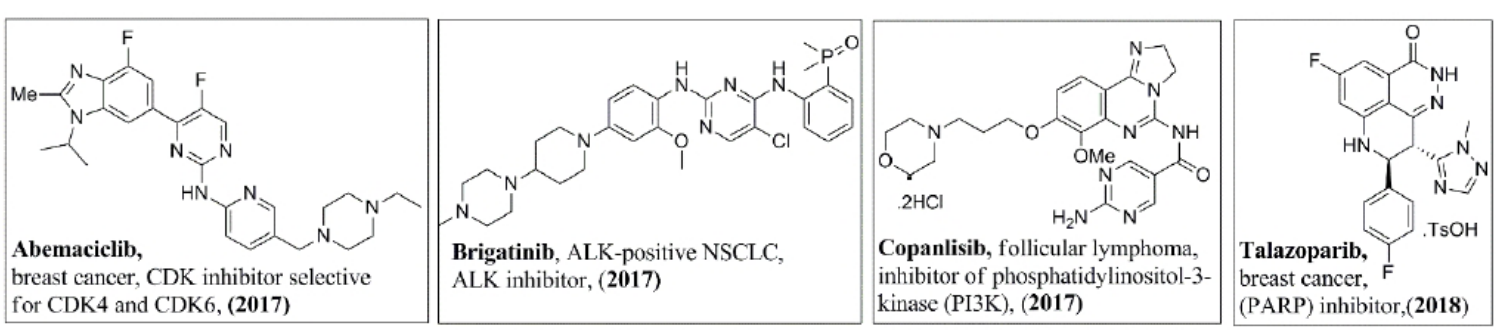

breast cancer,

(PARP) inhibitor, (2018)
Midostaurin, myeloid

leukemia, multi-targeted protein kinase inhibitor,(2017)

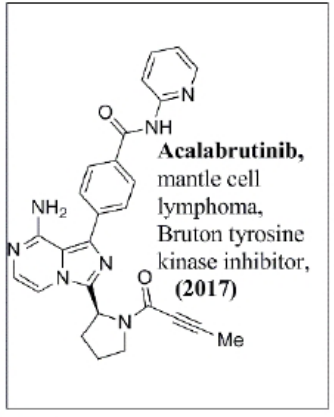

\begin{tabular}{|c|c|c|c|}
\hline $\begin{array}{l}\text { Ribociclib, } \\
\text { breast cancer, kinase inhibitor, (2017) }\end{array}$ & $\begin{array}{l}\text { Niraparib, Epithelial ovarian, } \\
\text { fallopian tube, or primary Peritoneal } \\
\text { cancer, poly-ADP ribose polymerase } \\
\text { inhibitor, (2017) }\end{array}$ & $\begin{array}{l}\text { Dacomitinib, } \\
\text { EGFR- } \\
\text { mutated } \\
\text { NSCLC, } \\
\text { EGFR } \\
\text { inhibitor, } \\
(\mathbf{2 0 1 8 )}\end{array}$ & $\begin{array}{l}\text { Larotrectinib, solid tumors, } \\
\text { kinase inhibitor,(2018) }\end{array}$ \\
\hline
\end{tabular}

Figure 6: The chemical structures of the approved drugs from 2017 to 2018. 

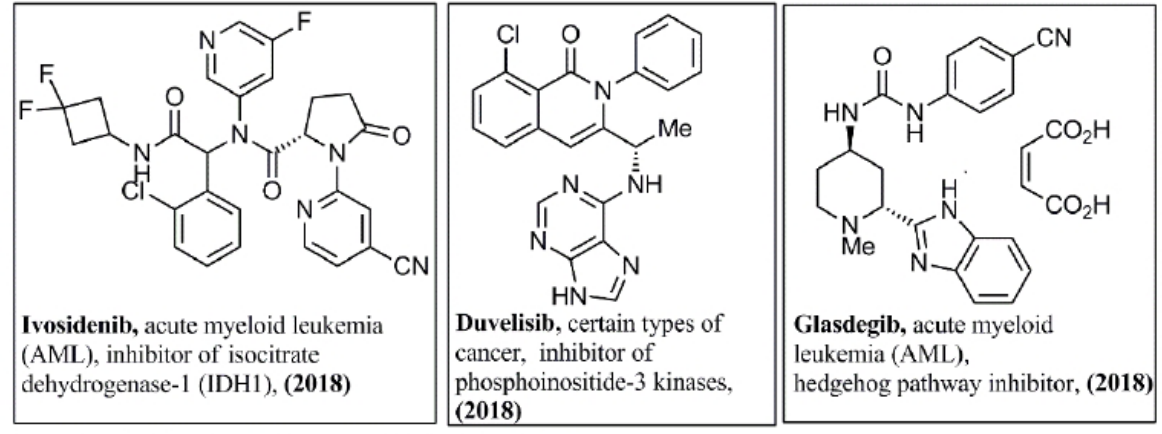

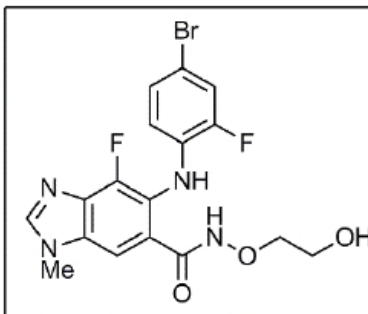

Binimetinib, metastatic melanoma, inhibits the activity of MEK1/2, (2018)
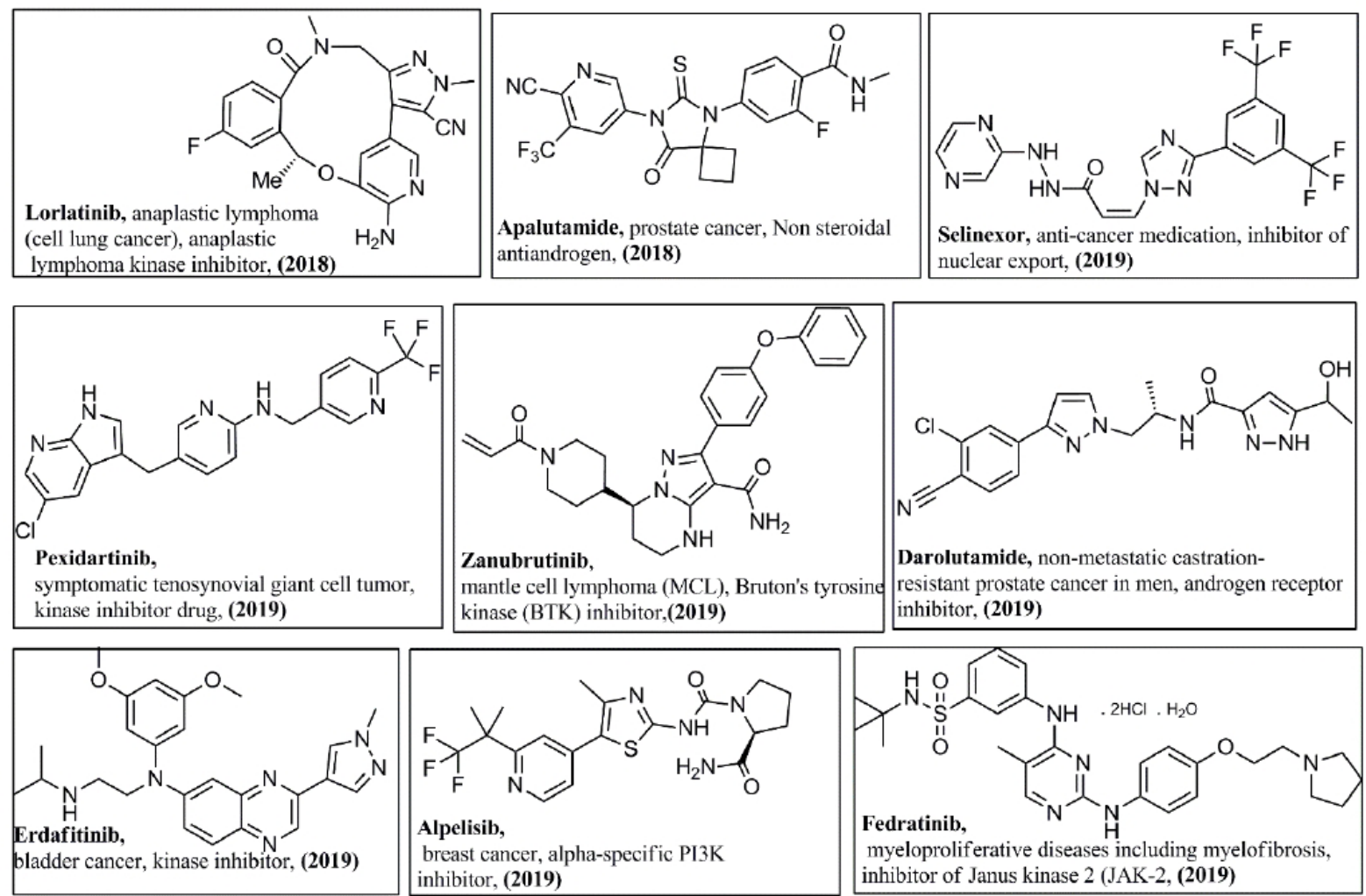

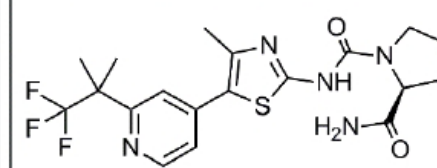

Alpelisib,

breast cancer, alpha-specific PI3K inhibitor, (2019)

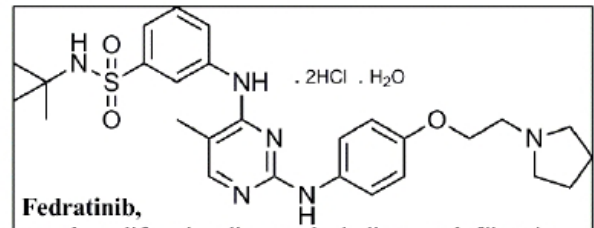

myeloproliferative diseases including myelofibrosis, inhibitor of Janus kinase 2 (JAK-2, (2019)

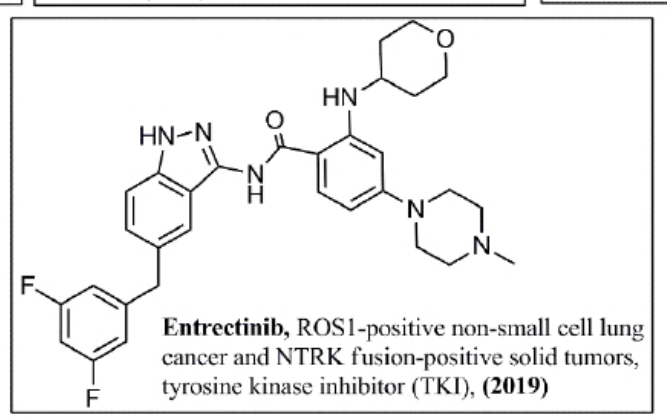

Figure 7: The chemical structures of the approved drugs from 2018 to 2019. 

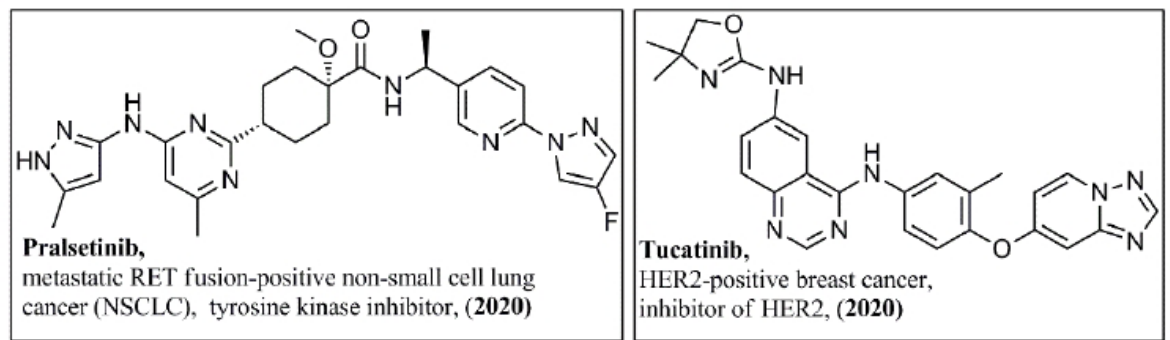

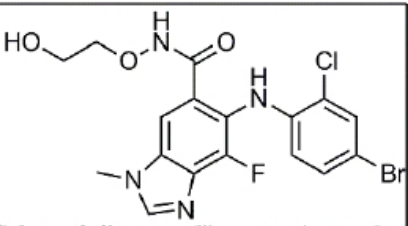

Selumetinib, neurofibromatosis type I (NF-1), inhibitor of the enzyme mitogen activated protein kinase (MAPK kinase or MEK) subtypes 1 and 2, (2020)
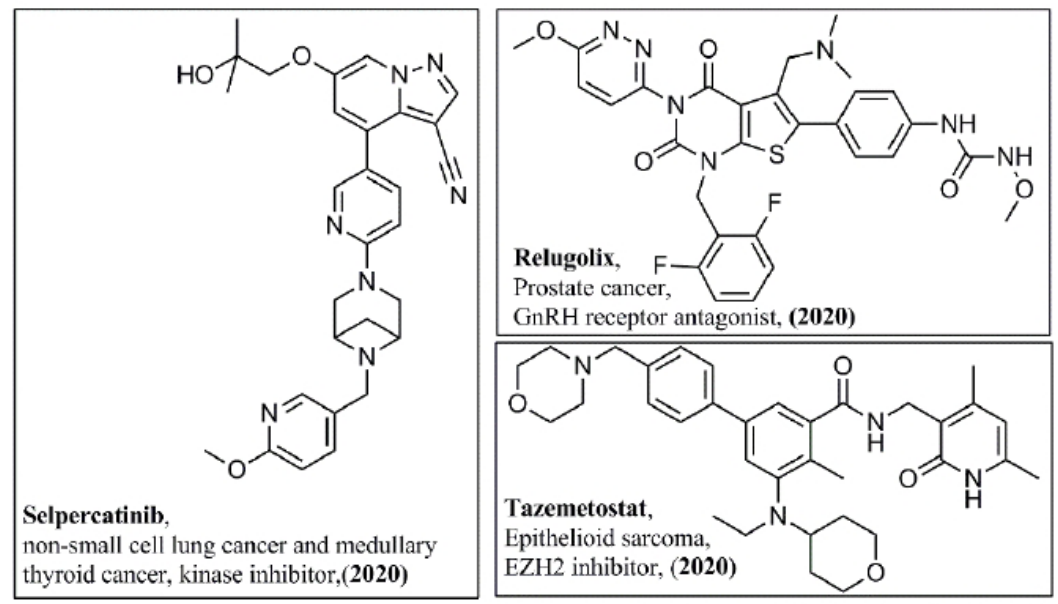

$\mathrm{H}_{3} \mathrm{C}-\mathrm{O}$

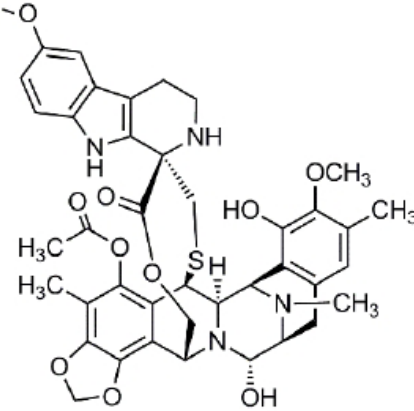

Lurbinectedin, metastatic small cell lung cancer (SCLC), inhibits the active transcription of the encoding genes, (2020)
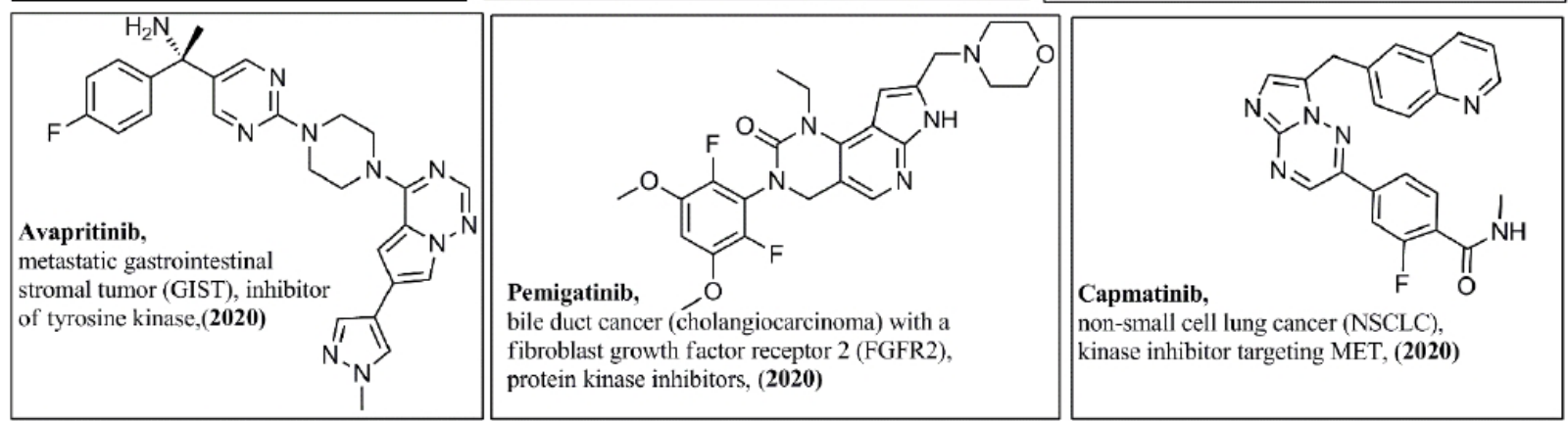

Figure 8: The chemical structures of the approved drugs in 2020.

\section{Kinase Inhibitors}

In the past decade, kinase inhibitors represent the majority of the FDA approved small molecules anticancer drugs. Most of these kinase inhibitors are tyrosine kinase inhibitors either to receptor kinases or to intracellular kinases.

\section{Tyrosine kinase inhibitors}

Receptor-linked tyrosine kinase (RTK) inhibitors: Receptor tyrosine kinases are cell surface receptors for various ligands. They have three domains; extracellular domain (ligand-binding domain, transmembrane domain and intracellular kinase-binding domain [4]. Some inhibitors of this class target a specific RTK, which, in turn, inhibit all the downstream signaling pathway. Osimertinib (2015) [5] and Alectinib (2015) [6], which specifically inhibit epidermal growth factor receptor (EGFR) and anaplastic lymphoma kinase (ALK), respectively, are indicated for non-small cell lung cancer
(NSCLC). In addition, Tucatinib (2020) targets breast cancer by specifically inhibiting human epidermal growth factor receptor 2 (HER2) [7]. On the other hand, other multi-kinase inhibitors inhibit more than one RTK which, accordingly, block the subsequent phosphorylation steps and signal transduction. Examples of multi-RTK inhibitors are Neratinib (2017) is indicated for breast cancer, where it inhibits EGFR, HER2 and human epidermal growth factor receptor 4 (HER4) [8,9]. Other examples of RTK inhibitors that are indicated for NSCLC are Crizotinib (2011) [10], Afatinib (2013) [11,12], Ceritinib (2014) [13], Brigatinib (2017) [14,15], Lorlatinib (2018) [16], Dacomitinib (2018) [17,18], Entrectinib (2019) [19], Pralsetinib (2020) [20], and Capmatinib (2020) [21]. For thyroid cancer, there are Vandetanib (2011) [22], Cabozantinib (2012) [23], Lenvatinib (2015) [24], and Selpercatinib (2020) [25], which targets lung cancer as well. Others include Axitinib (2012) for kidney cancer [26-28], Ponatinib hydrochloride (2012) 
[29,30] and Midostaurin (2017) for leukemia [31,32], Regorafenib (2012) for colorectal cancer [33,34], Larotrectinib (2018) for solid tumors [35], Avapritinib (2020) for gastrointestinal stromal tumor [32], Erdafitinib (2019) for urothelial carcinoma [36], Pexidartinib (2019) for Tenosynovial giant cell tumor [19] and finally Pemigatinib (2020) for cholangiocarcinoma [37].

Cytoplasmic or non-receptor tyrosine kinases (NRTK) inhibitors: The NRTK are crucial for intracellular signal transfer after ligand binding to the cell surface receptor. Many small molecules have been approved to target certain members of this class affecting various types of malignancies. Janus kinase (JAK) is one of the NRTK members that is inhibited by Ruxolitinib (2011) [38,39] and Fedratinib (2019) [40] for Myelofibrosis. Breakpoint cluster region protein- Abelson murine leukemia (Bcr-Abl) is a fusion protein produced from a chimeric gene and it can be targeted by Bosutinib (2012) for treating Philadelphia chromosome-positive $(\mathrm{Ph}+)$ chronic myelogenous leukemia [41]. Moreover, Bruton Tyrosine kinase (BTK) is a member of NRTK that can be inhibited by Ibrutinib (2013) [42,43], Acalabrutinib (2017) [44,45] and Zanubrutinib (2019) [46] for mantle cell lymphoma.

\section{Serine/threonine kinase inhibitors}

Serine/Threonine kinases are a class of proteins that are present at the initial point of signalling pathway at the cell surface or as an intracellular intermediate in the pathway. Phosphoinositide 3-kinases (PI3K) are vital members in the intracellular signal transduction pathways. Examples for PI3K inhibitors are Copanlisib (2017) for follicular lymphoma [47], Duvelisib (2018) [48] and Idelalisib (2014) [49] for chronic lymphocytic leukemia (CLL) or small lymphocytic leukemia (SLL) , Alpelisib (2019) for breast cancer [50]. Cyclin-dependent kinases (CDKs) are a group of serine/threonine kinases that regulate the cell cycle at different stages [51]. The approved drugs that can target CDKs are Palbociclib (2015) [52,53], Abemaciclib (2017) [54,55], and Ribociclib (2017) [55], which are indicated for breast cancer. v-raf murine sarcoma viral oncogene homolog B1 (BRAF) is a serine-threonine kinase and an intracellular component of the mitogen-activated protein kinase (MAPK) pathway. Examples of the drugs that target BRAF are Vemurafenib (2011) [56-58] and Dabrafenib (2013) [59] that are indicate ad for melanoma. Mitogen-activated protein kinase kinase (MAP2K) or also called MEK) is also a component of the MAPK pathway located downstream to BRAF. MEK is a different kinase since it is mixed threonine and tyrosine kinase [60]. Examples of the MEK inhibitors are Trametinib (2013) [61] and Cobimetinib (2015) [56] that are indicated for melanoma with mutated BRAF and Selumetinib (2020) that is approved to treat neurofibromatosis type 1 [62]. Furthermore, Binimetinib (2018) has been approved to treat metastatic melanoma in combination with Encorafenib (2018), a BRAF inhibitor $[63,64]$.

\section{Drugs Affecting Nucleic Acids}

\section{PARP inhibitors}

Poly-ADP ribose polymerase-1 (PARP) are a group of enzymes that play a vital role in recognizing and repairing the damaged DNA. The approved PARP inhibitors include Olaparib (2014) for Advanced ovarian cancer and metastatic breast cancer [65], Rucaparib (2016) for ovarian cancer [66], Niraparib (2017) for fallopian tube, epithelial ovarian or primary Peritoneal cancer [67], and Talazoparib (2018) for breast cancer [68].

\section{Alkylating agents}

These agents directly interact with the DNA in the minor groove and methylate the DNA at guanine bases in the $\mathrm{C}-\mathrm{G}$ rich region. Thus, they affect the transcription process and DNA repair mechanism [69]. Example of this class is Trabectedin (2015) that is indicated for Liposarcoma or leiomyosarcoma $[22,23,70]$. On the other hand, Lurbinectedin (2020) is indicated for small cell lung cancer (SCLC) and it exerts its action by binding to the guanine bases in the minor groove of the DNA forming adduct [71,72].

\section{Histone-modifying enzymes inhibitors}

Histone deacetylase (HDAC) enzyme regulates different DNA-linked processes via deacetylation of histone proteins to be accessible with other enzymes related to replication, transcription, repair, and chromatin remodeling. Inhibition of this enzyme results in growth inhibition and apoptosis induction [73]. Panobinostat (2015) is a HDAC inhibitor that is indicated for treating multiple myeloma in combination with other anticancer agents [74]. Belinostat (2014) is another HDAC inhibitor that is indicated for refractory peripheral T-cell lymphoma [75]. On the other hand, methyltransferase methylates the lysine and arginine residues in histone proteins, regulating the gene expression. Tazemetostat (2020) is a methyltransferase inhibitor that inhibits hypertrimethylation of histones and hence, preventing cancer cell dedifferentiation. It is indicated for treating epithelioid sarcoma $[76,77]$.

\section{Others}

The FDA approved a combination of trifluridine and tipiracil (2015) that targets the DNA of malignant cells. Trifluridine is a nucleoside analogue that is phosphorylated by thymidine kinase forming the active trifluoridine triphosphate. Then, the active form is incorporated in the DNA instead of thymidine bases, blocking DNA function, DNA synthesis and hence tumor cell proliferation. Tipiracil is a thymidine phosphorylase inhibitor, that Prevents the degradation of the active trifluridine. This combination is indicated for colorectal cancer [78-80]. 


\section{Antiandrogens}

Antiandrogens are drugs that oppose the effect of the male sex hormones, and they are indicated for castration-resistant prostate cancer therapy. Enzalutamide (2012) [8-83], Darolutamide (2019] and Apalutamide (2018) [84] are androgen receptor inhibitors, where they inhibit or block androgen binding to its receptor, which in turn, inhibits the subsequent signaling pathway and prevents cancer progression. Relugolix (2020) is approved for the treatment of advanced prostate cancer via androgen deprivation therapy (ADT). The mode of action of Relugolix depends on antagonizing the gonadotropin-releasing hormone (GnRH) receptor, which, consequently, reduces the testosterone level [85]. Furthermore, Abiraterone acetate (2011) inhibits the biosynthesis of testosterone and other androgen decreasing their serum level [86].

\section{Hedgehog Pathway Inhibitors}

Vismodegib (2012) [87,88] and Sonidegib (2015) [89] are inhibitors of the hedgehog pathway through inhibiting the transmembrane smoothened (smo) protein. They are indicated for the treatment of basal cell carcinoma. Moreover, Glasdegib (2018) is also an inhibitor of the hedgehog pathway via selective and potent binding to smo receptor. It is approved as anticancer drug against acute myeloid leukemia, in combination with cytarabine (a chemotherapeutic agent) $[90,91]$.

\section{Miscellaneous}

Malignant cell death could be addressed by targeting several molecular targets and herein some of the FDA approved drugs with various targets within the last decade. Apoptosis is the most important venue to get rid of the cancer cells by various antineoplastic drugs. Venetoclax (2016) induces the onset of apoptosis, and it is approved to treat acute myeloid leukemia, CLL or SLL. It inhibits the B cell CLL/lymphoma 2 (BCL-2) family, which, in turn, induces apoptosis of the malignant cells [92]. Ivosidenib (2018) is an isocitrate dehydrogenase-1 (IDH1) inhibitor that is indicated for acute myeloid leukemia [93]. Other drugs are designed to modulate the individual immune system such as Pomalidomide (2013) that is indicated for Multiple myeloma in combination with dexamethasone. It modulates the immune system via activation of the T-cells and natural killer (NK) as well as inhibiting proinflammatory cytokines [94]. Furthermore, the protein synthesis process could be targeted to prevent cancer progression by inhibiting the translation such as Omacetaxine mepesuccinate (2012) that is indicated for chronic myelogenous leukemia [95,96]. Moreover, Selinexor (2019) is indicated for multiple myeloma. It inhibits the nuclear transport via binding to and inhibiting exportin-1 (XPO1), a nuclear exporter protein. Consequently, the cells undergo cell cycle arrest and apoptotic cell death $[97,98]$. Finally, Carfilzomib (2012) is a proteasome inhibitor via inhibition of its chymotrypsin-like activity. It is indicated for treatment of refractory multiple myeloma [99].

\section{References}

1. Abdelsalam EA, Zaghary WA, Amin KM, Abou Taleb N A, Mekawey A A I, et al. (2019) Synthesis and in vitro anticancer evaluation of some fused indazoles, quinazolines and quinolines as potential EGFR inhibitors. Bioorganic Chemistry 89: 102985.

2. Jingchun Sun, Qiang Wei, Yubo Zhou, Jingqi Wang, Qi Liu, Hua Xu, et al. (2017) A systematic analysis of FDA-approved anticancer drugs. BMC Syst Biol 11(5): 1-17.

3. Dembic Z (2020) Antitumor drugs and their targets. Molecules 25(23): 5776.

4. Zhenfang Du, Christine M Lovly (2018) Mechanisms of receptor tyrosine kinase activation in cancer. Mol Cancer 17(1): 58

5. Lilan Yi, Junsheng Fan, Ruolan Qian, Peng Luo, Jian Zhang (2019) Efficacy and safety of osimertinib in treating EGFR-mutated advanced NSCLC: A meta-analysis. Int J Cancer 145(1): 284-294.

6. Junsheng Fan, Zengfei Xia, Xiaoli Zhang, Yuqing Chen, Ruolan Qian et al. (2018) The efficacy and safety of alectinib in the treatment of ALK+ NSCLC: A systematic review and meta-analysis. Onco Targets Ther 11: 1105-1115.

7. Lara Ulrich, Alicia F C Okines (2021) Treating Advanced Unresectable or Metastatic HER2-Positive Breast Cancer: A Spotlight on Tucatinib. Breast Cancer: Targets and Therapy 13: 361-381.

8. Rutugandha Paranjpe, Dima Basatneh, Gabriel Tao, Carmine De Angelis, Sobia Noormohammed, et al. (2019) Neratinib in HER2-Positive Breast Cancer Patients. Annals of Pharmacotherapy 53(6): 612-620.

9. Chan A (2016) Neratinib in HER-2-positive breast cancer: Results to date and clinical usefulness. Ther Adv Med Oncol 8(5): 339-350.

10. Arvind Sahu, Kumar Prabhash, Vanita Noronha, Amit Joshi, Saral Desai (2013) Crizotinib: A comprehensive review. South Asian J Cancer 2(2): 91.

11. James Chih Hsin Yang, Martin Schuler, Sanjay Popat, Satoru Miura, Simon Heeke, et al. (2020) Afatinib for the Treatment of NSCLC Harboring Uncommon EGFR Mutations: A Database of 693 Cases. Journal of Thorac Oncol 15(5): 803-815.

12. Pasqualina Giordano, Anna Manzo, Agnese Montanino, Raffaele Costanzo, Claudia Sandomenico, et al. (2016) Afatinib: An overview of its clinical development in non-small-cell lung cancer and other tumors. Crit Rev Oncol Hematol 97: 143-151.

13. John Kaczmar, Ranee Mehra (2015) The efficacy of ceritinib in patients with ALK-positive non-small cell lung cancer. Ther Adv Respir Dis 9(5): 236-241.

14. Stewart Umbela, Shahinaz Ghacha, Revika Matuknauth, Stacey Gause, Shrijana Joshee, et al. (2019) Brigatinib: New-generation ALK inhibitor for nonsmall cell lung cancer. Curr Probl Cancer 43(6): 100477.

15. Silky Bedi, Shah A Khan, Majed M AbuKhader, Perwez Alam, Nasir A Siddiqui, et al. (2018) A comprehensive review on Brigatinib- A wonder drug for targeted cancer therapy in Non-Small Cell Lung Cancer A comprehensive review on Brigatinib - A wonder drug for targeted cancer therapy in non-small cell lung cancer. Saudi Pharm J 26(6): 755763.

16. Alice T Shaw, Enriqueta Felip, Todd M Bauer, Benjamin Besse , Alejandro Navarro, et al. (2017) Lorlatinib in non-small-cell lung cancer with ALK or ROS1 rearrangement: an international, multicentre, open-label, single-arm first-in-man phase 1 trial. The Lancet Oncology 18(12): 1590-1599. 
17. Daniele Lavacchi, Francesca Mazzoni, Giuseppe Giaccone (2019) Clinical evaluation of dacomitinib for the treatment of metastatic non-small cell lung cancer (NSCLC): Current perspectives. Drug Des Devel Ther 13 3187-3198.

18. Cecilia Bergonzini, Alessandro Leonetti, Marcello Tiseo, Elisa Giovannetti, Godefridus J Peters (2020) Is there a role for dacomitinib, a second- generation irreversible inhibitor of the epidermal- growth factor receptor tyrosine kinase, in advanced non-small cell lung cancer? Expert Opinion Pharmacother 21(11): 1287-1298.

19. Brooke Benner, Logan Good, Dionisia Quiroga, Thomas E Schultz, Mahmoud Kassem, et al. (2020) Pexidartinib, a novel small molecule csf-1r inhibitor in use for tenosynovial giant cell tumor: A systematic review of pre-clinical and clinical development. Drug Des Devel Ther 14: 1693-1704.

20. Markham A (2020) Pralsetinib: First Approval. Drugs 80(17): 18651870.

21. Johan Filip Vansteenkiste, Charlotte Van De Kerkhove, Els Wauters, Pierre Van Mol (2019) Capmatinib for the treatment of non-small cell lung cancer. Expert Rev Anticancer Ther 19(8): 659-671.

22. Maryse Brassard, Genevieve Rondeau (2012) Role of vandetanib in the management of medullary thyroid cancer. Biologics: Targets and Therapy 6: 59-66.

23. Nizar M Tannir, Gisela Schwab, Viktor Grunwald (2017) Cabozantinib: an Active Novel Multikinase Inhibitor in Renal Cell Carcinoma. Curr Oncol Rep 19(2): 1-9.

24. Shunji Takahashi, Naomi Kiyota, Tomoko Yamazaki, Naoko Chayahara, Kenji Nakano, et al. (2019) A Phase II study of the safety and efficacy of lenvatinib in patients with advanced thyroid cancer. Future Oncol 15(7): 717-726.

25. Oliver Illini, Maximilian Johannes Hochmair, Hannah Fabikan, Christoph Weinlinger , Amanda Tufman, et al. (2021) Selpercatinib in RET fusion positive non-small-cell lung cancer (SIREN): a retrospective analysis of patients treated through an access program. Ther Adv Med Oncol 13: 17588359211019675 .

26. Revill P, Mealy N, Bayes M, Bozzo J, Serradell N, et al. (2007) Axitinib: VEGFR/PDGFR tyrosine kinase inhibitor antiangiogenic agent. Drugs of the Future 32(5): 389-398.

27. Hiral Parekh, Julianne Griswold, Brian Rini (2016) Axitinib for the treatment of metastatic renal cell carcinoma. Future Oncol 12(3): 303311.

28. Marine Gross Goupil, Louis Francois, Amandine Quivy, Alain Ravaud (2013) Axitinib: A review of its safety and efficacy in the treatment of adults with advanced renal cell carcinoma. Clin Meda Insights Oncol 7: 269-277.

29. Jain H, Thorat J, Sengar M, Dubey A (2019) Ponatinib: A drug review. Cancer Research, Statistics, and Treatment 2(2): 190.

30. Fiona H Tan, Tracy L Putoczki, Stanley S Stylli, Rodney B Luwor (2019) Ponatinib: A novel multi-tyrosine kinase inhibitor against human malignancies. Onco Targets Ther 12: 635-645.

31. Molly Megan Gallogly, Hillard M Lazarus (2016) Midostaurin: An emerging treatment for acute myeloid leukemia patients. J Blood Med 7: 73-83.

32. Johannes Lubke, Nicole Naumann, Sebastian Kluger, Juliana Schwaab, Georgia Metzgeroth, et al. (2019) Inhibitory effects of midostaurin and avapritinib on myeloid progenitors derived from patients with KIT D816V positive advanced systemic mastocytosis. Leukemia 33(5): 11951205.

33. Saravanan K Krishnamoorthy, Valerie Relias, Sunit Sebastian, Vijay Jayaraman , Muhammad Wasif Saif 5 (2015) Management of regorafenibrelated toxicities: A review. Therap Adv Gastroenterol 8(5): 285-297.
34. Jean Baptiste Rey, Vincent Launay Vacher, Christophe Tournigand (2015) Regorafenib as a single-agent in the treatment of patients with gastrointestinal tumors: an overview for pharmacists. Targeted Oncol 10(2): 199-213.

35. D S Hong, T M Bauer, J J Lee, A Dowlati, M S Brose, et al. (2019) Larotrectinib in adult patients with solid tumours: A multi-centre, openlabel, phase i dose-escalation study. Ann Oncol 30(2): 325-331.

36. Loriot Y, Necchi A, Park SH, Garcia Donas J, Huddart, R, et al. (2019) Erdafitinib in Locally Advanced or Metastatic Urothelial Carcinoma. New England Journal of Medicine 381(4): 338-348.

37. Valeria Merz, Camilla Zecchetto, Davide Melisi (2021) Pemigatinib, a potent inhibitor of FGFRs for the treatment of cholangiocarcinoma. Future Oncol 17(4): 389-402.

38. Mesa RA (2010) Ruxolitinib, a selective JAK1 and JAK2 inhibitor for the treatment of myeloproliferative neoplasms and psoriasis. IDrugs 13(6): 394-403.

39. Kris Vaddi, Srdan Verstovsek, Jean Jacques Kiladjian (2016) Ruxolitinib: a targeted treatment option for patients with polycythemia vera. Blood Lymphat Cancer: Targets and Therapy 6: 7-19.

40. Moshe Talpaz, Jean acques Kiladjian (2021) Fedratinib, a newly approved treatment for patients with myeloproliferative neoplasm-associated myelofibrosis. Leukemia 35(1): 1-17.

41. Adil I Daud, Smitha S Krishnamurthi, Mansoor N Saleh, Barbara J Gitlitz, Mitesh J Borad, et al. (2012) Phase I study of bosutinib, a Src/Abl tyrosine kinase inhibitor, administered to patients with advanced solid tumors. Clin Cancer Res 18 (4): 1092-1100.

42. Gretchen Anne McNally, Jennifer M Long, Lynne R Brophy, Maria R Badillo (2015) Ibrutinib: Implications for Use in the Treatment of Mantle Cell Lymphoma and Chronic Lymphocytic Leukemia. J Adv Pract Oncol 6 (5): 420-431.

43. Julio C Chavez, Eva Sahakian, Javier Pinilla Ibarz (2013) Ibrutinib: An evidence-based review of its potential in the treatment of advanced chronic lymphocytic leukemia. Core Evid 8: 37-45.

44. Wojciech Jurczak, Monika Dlugosz Danecka, Michael Wang (2019) Acalabrutinib for adults with mantle cell lymphoma. Expert Rev Clin Pharmacol 12(3): 179-187.

45. Tjeerd Barf, Todd Covey, Raquel Izumi, Bas van de Kar, Michael Gulrajani, et al. (2017) Acalabrutinib (ACP-196): A covalent Bruton tyrosine kinase inhibitor with a differentiated selectivity and in vivo potency profile. J Pharmacol Exp Ther 363(2): 240-252.

46. Praveen Ramakrishnan Geethakumari, Farrukh Awan (2020) An evaluation of zanubrutinib, a BTK inhibitor, for the treatment of chronic lymphocytic leukemia. Expert Rev Hematol 13(10): 1039-1046.

47. Ayushi F Chauhan, Bruce D Cheson (2021) Copanlisib in the treatment of relapsed follicular lymphoma: Utility and experience from the clinic. Cancer Manag Res 13: 677-692.

48. Ian W Flinn, Susan O Brien, Brad Kahl, Manish Patel, Yasuhiro Oki et al. (2018) Duvelisib, a novel oral dual inhibitor of PI3K-d,g, is clinically active in advanced hematologic malignancies. Blood 131(8): 877-887.

49. Maliha Khan, Areeba Saif, Steven Sandler, Aibek E Mirrakhimov (2014) Idelalisib for the Treatment of Chronic Lymphocytic Leukemia. ISRN Oncol 2014: 931858.

50. Avan J Armaghani, Hyo Sook Han (2020) Alpelisib in the treatment of breast cancer: A short review on the emerging clinical data. Breast Cancer(Dove Med Press) 12: 251-258.

51. Sameh H Mohamed, Hossam R Elgiushy, Heba Taha, Sherif F Hammad, Nageh A Abou Taleb, et al. (2020) An investigative study of antitumor properties of a novel thiazolo[4,5-d]pyrimidine small molecule revealing superior antitumor activity with CDK1 selectivity and potent pro-apoptotic properties. Bioorg Med Chem 28(17): 115633. 
52. Karen A Cadoo, Ayca Gucalp, Tiffany A Traina (2014) Palbociclib: An evidence-based review of its potential in the treatment of breast cancer Breast Cancer: (Dove Med Press) 6:123-133.

53. Richard S Finn, John P Crown, Johannes Ettl, Marcus Schmidt, Igor M Bondarenko, et al. (2016) Efficacy and safety of palbociclib in combination with letrozole as first-line treatment of ER-positive, HER2negative, advanced breast cancer: Expanded analyses of subgroups from the randomized pivotal trial PALOMA-1/TRIO-18. Breast Cancer Res 18(1): 67

54. Amita Patnaik, Lee S Rosen, Sara M Tolaney, Anthony W Tolcher, Jonathan W Goldman, et al. (2016) Efficacy and safety of Abemaciclib, an inhibitor of CDK4 and CDK6, for patients with breast cancer, non-small cell lung cancer, and other solid tumors. Cancer Discov 6(7): 740-753.

55. Rossana Roncato, Jacopo Angelini, Arianna Pani, Erika Cecchin, Andrea Sartore Bianchi, et al. (2020) Cdk4/6 inhibitors in breast cancer treatment: Potential interactions with drug, gene, and pathophysiological conditions. Int J Mol Sci 21(17): 6350.

56. Boespflug A, Thomas L (2016) Cobimetinib and vemurafenib for the treatment of melanoma. Expert Opin Pharmacother 17(7): 1005-1011.

57. Abhisek Swaika, Jennifer A Crozier, Richard W Joseph (2014) Vemurafenib: An evidence-based review of its clinical utility in the treatment of metastatic melanoma. Drug Des Devel Ther 8: 775-787.

58. Gideon Bollag, James Tsai, Jiazhong Zhang, Chao Zhang, et al. (2012) Vemurafenib: The first drug approved for BRAF-mutant cancer. Nat Rev Drug Discov 11(11): 873-886.

59. Victoria G Atkinson, Pietro Quaglino, Massimo Aglietta, Michele Del Vecchio, et al. (2021) A Retrospective Analysis of Dabrafenib and / or Dabrafenib Plus Trametinib Combination in Patients with Metastatic Melanoma to Characterize Patients with Long-Term Benefit in the Individual Patient Program ( DESCRIBE III ). Cancers (Basel) 13(10): 2466.

60. Montagut C, Settleman J (2009) Targeting the RAF-MEK-ERK pathway in cancer therapy. Cancer Lett 283(2): 125-134.

61. Lugowska I, Kosela Paterczyk H, Kozak K, Rutkowski P (2015) Trametinib: A MEK inhibitor for management of metastatic melanoma. Onco Targets Ther 8: 2251-2259.

62. Eva Dombi, Andrea Baldwin, Leigh J Marcus, Michael J Fisher, Brian Weiss et al. (2016) Activity of Selumetinib in Neurofibromatosis Type 1-Related Plexiform Neurofibromas. N Engl J Med 375(26): 2550-2560.

63. Koelblinger P, Dornbierer J, Dummer R (2017) A review of binimetinib for the treatment of mutant cutaneous melanoma. Future Oncol 13(20): $1-12$.

64. Sun J, Zager JS, Eroglu Z (2018) Encorafenib/binimetinib for the treatment of BRAF-mutant advanced, unresectable, or metastatic melanoma: Design, development, and potential place in therapy. Onco Targets The 11: 9081-9089.

65. Soiza RL, Donaldson AIC, Myint PK (2018) Vaccine against arteriosclerosis: an update. Therapeutic Advances in Vaccines 9(6): 259261.

66. L E Dockery, C C Gunderson, K N Moore (2017) Rucaparib: The past, present, and future of a newly approved PARP inhibitor for ovarian cancer. Onco Targets Ther 10: 3029-3037.

67. Sisay M, Edessa D (2017) PARP inhibitors as potential therapeutic agents for various cancers: focus on niraparib and its first global approval for maintenance therapy of gynecologic cancers. Gynecol Oncol Res Pract $4: 18$

68. Stergios Boussios , Charlotte Abson, Michele Moschetta, Elie Rassy, Afroditi Karathanasi, et al. (2020) Poly (ADP-Ribose) Polymerase Inhibitors: Talazoparib in Ovarian Cancer and Beyond. Drugs R D 20(2): 55-73.
69. Larissa Costa de Almeida, Felipe Antunes Calil, Joao Agostinho Machado Neto, Leticia Veras Costa Lotufo (2021) DNA damaging agents and DNA repair: From carcinogenesis to cancer therapy. Cancer Genet 252-253: 6-24.

70. Katherine A Thornton (2010) Trabectedin: The evidence for its place in therapy in the treatment of soft tissue sarcoma. Core Evid 4: 191-198.

71. Sonia Singh, Adnan A Jaigirdar, Flora Mulkey, Joyce Cheng, Salaheldin $S$ Hamed, et al. (2021) FDA approval summary: Lurbinectedin for the treatment of metastatic small cell lung cancer. Clin Cancer Res 27(9): 2378-2382.

72. Kauffmann Guerrero D, Huber RM (2020) Orphan drugs in development for the treatment of small-cell lung cancer: Emerging data on lurbinectedin. Lung Cancer(Auck) 11: 27-31.

73. Gallinari P, Di Marco S, Jones P, Pallaoro M, Steinkühler C (2007) HDACs, histone deacetylation and gene transcription: From molecular biology to cancer therapeutics. Cell Research 17(3): 195-211.

74. Eleutherakis Papaiakovou E, Kanellias N, Kastritis E, Gavriatopoulou M, Terpos E, et al. (2020) Efficacy of Panobinostat for the Treatment of Multiple Myeloma. J Oncol 2020.

75. Zhao Jennifer C, Jaszczur Sara Mohamed, Afifi Salma, Foss Francine (2020) Pralatrexate injection for the treatment of patients with relapsed or refractory peripheral T-cell lymphoma. Expert Rev Hematol 13(6): 577-583.

76. Morschhauser F, Tilly H, Chaidos, A, Phillips TJ, Ribrag V, et al. (2019) Phase 2 Multicenter Study of Tazemetostat, an EZH2 Inhibitor, in Patients with Relapsed or Refractory Follicular Lymphoma. Blood 134: 123-123.

77. Ghazal Tansir, Sameer Rastogi, Shamim Ahmed Shamim, Adarsh Barwad (2021) Early clinical and metabolic response to tazemetostat in advanced relapsed INI1 negative epithelioid sarcoma. Future Sci OA 7(4): FSO675

78. Jean Baptiste Bachet, Lucjan Wyrwicz, Timothy Price, Chiara Cremolini, Jean Marc Phelip, et al. (2020) Safety, efficacy and patient-reported outcomes with trifluridine/tipiracil in pretreated metastatic colorectal cancer: results of the PRECONNECT study. ESMO Open 5(3): 000698.

79. Johannes J M Kwakman, G Vink, J H Vestjens, L V Beerepoot, J W de Groot, et al. (2018) Feasibility and effectiveness of trifluridine/tipiracil in metastatic colorectal cancer: real-life data from The Netherlands. Int J Clin Oncol 23(3): 482-489.

80. Burness CB, Duggan ST (2016) Trifluridine/Tipiracil: A Review in Metastatic Colorectal Cancer. Drugs 76(14): 1393-1402.

81. Ian D Davis, Andrew J Martin, Martin R Stockler, Stephen Begbie, Kim N Chi, et al. (2019) Enzalutamide with Standard First-Line Therapy in Metastatic Prostate Cancer. N Engl J Med 381(2): 121-131.

82. Giuseppe Cicero, Rossella DE Luca, Patrizia Dorangricchia, Francesco Dieli (2017) The clinical efficacy of enzalutamide in metastatic prostate cancer: Prospective single-center study. Anticancer Res 37(3): 14751480

83. Golshayan AR, Antonarakis ES (2013) Enzalutamide: An evidence-based review of its usein the treatment of prostate cancer. Core Evid 8: 27-35.

84. Keiichiro Mori, Hadi Mostafaei, Benjamin Pradere, Reza Sari Motlagh, Fahad Quhal, et al. (2020) Apalutamide, enzalutamide, and darolutamide for non-metastatic castration-resistant prostate cancer: a systematic review and network meta-analysis. Int J Clin Oncol 25(11): 1892-1900.

85. Saad F, Shore, ND (2021) Relugolix: a novel androgen deprivation therapy for management of patients with advanced prostate cancer. Ther Adv Med Oncol 13: 1-12.

86. Bram L T Ramaekers, Rob Riemsma, Florian Tomini, Thea van Asselt, Sohan Deshpande, et al. (2016) Abiraterone Acetate for the Treatment of Chemotherapy-Nar Metastatic Castration-Resistant Prostate Cancer : 
An Evidence Review Group Perspective of an NICE Single Technology Appraisal. Pharmacoeconomics 35(2):191-202

87. Aleksandar Sekulic 1, Michael R Migden, Anthony E Oro, Luc Dirix, Karl D Lewis, et al. (2012) Efficacy and Safety of Vismodegib in Advanced BasalCell Carcinoma. N Engl J Med 366(23): 2171-2179.

88. Macha MA, Batra SK, Ganti AK (2013) Profile of vismodegib and its potential in the treatment of advanced basal cell carcinoma. Cancer Management and Research 5(1): 197-203.

89. Sachin Jain, Ruolan Song, Jingwu Xie (2017) Sonidegib: Mechanism of action, pharmacology, and clinical utility for advanced basal cell carcinomas. Onco Targets Ther10: 1645-1653.

90. Parvesh Chaudhry, Mohan Singh, Timothy J Triche, Monica Guzman, Akil A Merchant (2017) GLI3 repressor determines Hedgehog pathway activation and is required for response to SMO antagonist glasdegib in AML. Blood 129(26): 3465-3475.

91. US Food and Drug Administration (2020) FDA approves glasdegib for AML in adults age 75 or older or who have comorbidities (2018).

92. Toby A Eyre, Lindsey E Roeker, Christopher P Fox, Satyen H Gohill, Renata Walewska, et al. (2020) The efficacy and safety of venetoclax therapy in elderly patients with relapsed, refractory chronic lymphocytic leukaemia. British Journal of Haematology 188(6): 918-923.

93. Pasquier F, Chahine C, Marzac C, de Botton S (2020) Ivosidenib for the treatment of relapsed or refractory acute myeloid leukemia with an IDH1 mutation. Expert Review of Precision Medicine and Drug Development 5(6): 429-438.

94. Evangelos Terpos, Nikolaos Kanellias, Dimitrios Christoulas, Efstathios Kastritis, Meletios A Dimopoulos (2013) Pomalidomide: A novel drug to treat relapsed and refractory multiple myeloma. Onco Targets Ther 6: 531-538.

95. V Narayanan, J A Gutman D A Pollyea, A Jimeno (2013) Omacetaxine mepesuccinate for the treatment of chronic myeloid leukemia. Drugs of Today 49(7): 447-456.

96. Omar Al Ustwani, Elizabeth A Griffiths, Eunice S Wang, Meir Wetzler (2014) Omacetaxine mepesuccinate in chronic myeloid leukemia. Expert Opinion on Pharmacother 15(16): 2397-2405.

97. Joshua Richter, Deepu Madduri, Shambavi Richard, Ajai Chari (2020) Selinexor in relapsed/refractory multiple myeloma. Ther Adv in Hematol 11: 2040620720930629

98. Ujjawal H Gandhi, William Senapedis, Erkan Baloglu, Thaddeus J Unger Ajai Chari, et al. (2018) Clinical Implications of Targeting XP01-mediated Nuclear Export in Multiple Myeloma. Clinical Lymphoma, Myeloma and Leukemia 18(5): 335-345.

99. Karel Fostier, Ann De Becker, Rik Schots (2012) Carfilzomib: A novel treatment in relapsed and refractory multiple myeloma. Onco Targets Ther 5: 237-244. 